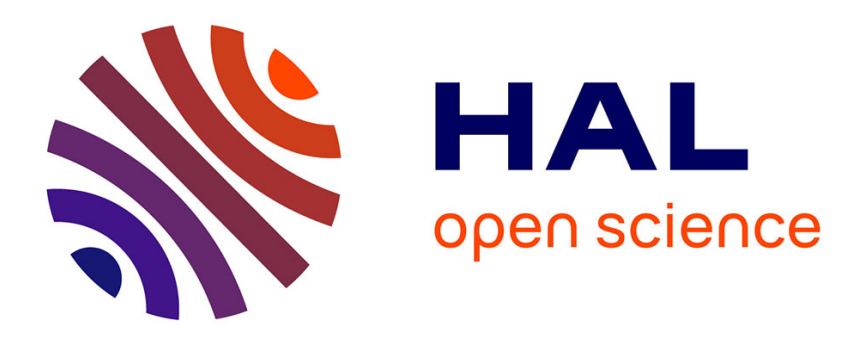

\title{
Molecular dynamics simulation of the first stages of the cavitation process in amorphous polymers \\ Sixou Bruno
}

\section{To cite this version:}

Sixou Bruno. Molecular dynamics simulation of the first stages of the cavitation process in amorphous polymers. Molecular Simulation, 2007, 33 (12), pp.965-973. 10.1080/08927020701502057 . hal00515012

\section{HAL Id: hal-00515012 \\ https://hal.science/hal-00515012}

Submitted on 4 Sep 2010

HAL is a multi-disciplinary open access archive for the deposit and dissemination of scientific research documents, whether they are published or not. The documents may come from teaching and research institutions in France or abroad, or from public or private research centers.
L'archive ouverte pluridisciplinaire HAL, est destinée au dépôt et à la diffusion de documents scientifiques de niveau recherche, publiés ou non, émanant des établissements d'enseignement et de recherche français ou étrangers, des laboratoires publics ou privés. 


\section{Molecular Simulation}

Journal of

Experimental Nanoscience

Taylor \& Francis

Taglor \& Francis Group

\section{Molecular dynamics simulation of the first stages of the cavitation process in amorphous polymers}

\begin{tabular}{|c|c|}
\hline Journal: & Molecular Simulation/Journal of Experimental Nanoscience \\
\hline Manuscript ID: & GMOS-2007-0060 \\
\hline Journal: & Molecular Simulation \\
\hline $\begin{array}{r}\text { Date Submitted by the } \\
\text { Author: }\end{array}$ & 23-May-2007 \\
\hline Complete List of Authors: & Bruno, Sixou; GEMPPM-MATEIS, INSA Lyon \\
\hline Keywords: & cavitation, polymers, molecular dynamics \\
\hline
\end{tabular}

\section{SCHOLARONE ${ }^{\text {m }}$ \\ Manuscripts}




\title{
Molecular dynamics simulation of the first stages of the
}

\section{cavitation process in amorphous polymers}

\author{
B.SIXOU, MATEIS, INSA de Lyon \\ INSA, MATEIS, Bt.B.Pascal, UMR CNRS 5510, 20 av.Einstein, 69621, Villeurbanne Cedex, \\ France \\ Email : Bruno.Sixou@insa-lyon.fr
}

\begin{abstract}
The first stages of the cavitation process in amorphous polymers submitted to an hydrostatic deformation in the glassy state are studied with coarse grain molecular dynamics simulations for various intermolecular interactions strengths and flexible and semi-flexible chains. For strong intermolecular interactions, the cavitation process is highly localized and the holes have a marked spherical symmetry. The cavitation regions are more diffuse for weaker intermolecular interactions or when the chain stiffness is increased. The mean Voronoï polyhedra volume and the disorder inside the polymer increase until the stress peak observed below the glass transition. High mobility regions are present before the stress peak that may act as nucleation sites for the cavitation process. The localization of these high mobility zones is enhanced for strong intermolecular interactions or a low chain rigidity. Moreover, the velocity fluctuations are more marked in the vicinity of the holes. For strong intermolecular interactions, the holes are not randomly distributed throughout the system and the nucleation of cavities upon deformation occurs preferentially near the chain ends of the polymer.
\end{abstract}

Keywords: Cavitation, polymers, molecular dynamics 


\section{Introduction}

It is well known that the failure of glassy polymers under external stresses occurs either through shear deformation or through crazing. In general, strong hydrostatic stresses favour crazing. Yet, no clear consensus exists about the stress state required to initiate crazing. Several aspects of crazing and cavitation physics have already been addressed with simulations in previous papers. A number of cavitation criteria have been formulated in order to predict the conditions under which a material will cavitate, such as the one of Rottler and Robbins [1]. It is well established that below $\mathrm{T}_{\mathrm{g}}$ an elastic behaviour is followed by a stress peak, which is generally associated to large holes. Yet, there is still little understanding about the conditions and about the mechanisms underlying the formation of the voids [2]. Nevertheless, a better understanding of the molecular origin of the mechanical properties of amorphous polymers has been achieved recently and recent simulations have emphasized the fact that these properties are closely related to the chains molecular mobility. It has been shown by Capaldi et al. [3] that the plastic deformation of glassy amorphous polymers is related to an enhanced mobility. Significant increases in torsional transition rates are observed during the deformation prior to and just beyond the yield point. The mobility may therefore also be a key factor affecting the cavitation process. On the other hand, as demonstrated by numerous experimental studies and computer simulations, the dynamics in amorphous polymers below $\mathrm{T}_{\mathrm{g}}$ is very heterogeneous [4-9]. Dynamical heterogeneities are defined as the the fact that some atoms or dihedrals exhibit much faster than average dynamics, while the others exhibit much slower motions. An increase of the heterogeneity in the rate of conformational transitions was obtained with decreasing temperature in amorphous polymers. Yet, there is no clear correlation between the local packing environment and the mobility 
differences observed. Moreover, some molecular dynamics simulations have suggested that the deformation process is associated with local stress, local deformation and mobility heterogeneities $[10,11,12]$. Therefore, dynamical and deformation heterogeneities may play a major role during the first stages of the cavitation process.

In this work, we present coarse grain molecular dynamics simulations of amorphous polymers in order to study the first steps of the cavitation process. In the coarse grain method, several monomers are replaced by one bead and polymers are modelled on a scale that takes into account Van der Waals and covalent interactions without specific reference to the chemical details. Much of the physics of bulk amorphous polymers is independent of the chemical details and arises from the connectivity of the beads. This type of simulation has thus provided much insight into the universal aspects of the structure and of the dynamics in amorphous or semi-crystalline polymers [13]. In this paper, we use this type of molecular dynamics simulations in order to study in detail the first stages of the cavitation process and the molecular mechanisms at the origin of this phenomenon. The distributions of occupied and unoccupied volumes are examined with hard sphere free volume methods and a Voronoï tessellation. We intend to clarify the relationships between the free volume increase and the molecular mobility evolution during the first steps of the cavitation process. We investigate the effect of the chain rigidity and of the strength of the intermolecular interactions on the free volume shape and distribution and on the mobility heterogeneities associated to the nucleation and to the development of the cavities. The comparison of the results gives interesting qualitative results into the cavitation phenomenon. We will also show a correlation between the chain ends and the holes that appear during the first stages of the deformation process. This paper is structured as follows. First, we present the simulations details. This first section is followed by results and discussion. We will summarize our findings in the conclusion section. 


\section{Simulation methodology}

We have performed molecular dynamics simulations of a standard coarse-grained polymer model. This model incorporates covalent backbone bonds, excluded-volume interactions and the chain stiffness [14-16]. All monomers have the same mass $\mathrm{m}$. The Van der Waals interactions between beads separated by a distance $r$ are modelled with a truncated and shifted Lennard-Jones potential:

$V_{L J}(r)=4 \varepsilon\left[\left(\frac{\sigma}{r}\right)^{12}-\left(\frac{\sigma}{r}\right)^{6}\right]-4 \varepsilon\left[\left(\frac{\sigma}{r_{c}}\right)^{12}-\left(\frac{\sigma}{r_{c}}\right)^{6}\right]$

for $\mathrm{r}<\mathrm{r}_{\mathrm{c}}$.

The potential is zero if the separation $r$ between two beads exceeds a cutoff radius $r_{c}$. The parameters $\varepsilon$ and $\sigma$ are characteristic energy and length scales, respectively. The simulations have been performed in reduced units, $m=1, \sigma=1, \varepsilon=1$. The characteristic time scale of the simulations is $\tau_{L J}=\sqrt{\frac{m \sigma^{2}}{\varepsilon}}=1$.

The Finitely Extensible Nonlinear Elastic (FENE) potential was used for covalent bonds between the successive beads [14-16]:

$V(r)=-\frac{k R_{0}{ }^{2}}{2} \ln \left(1-\frac{r^{2}}{R_{0}{ }^{2}}\right)$

with the values $\mathrm{R}_{0}=1.5 \sigma, \mathrm{k}=30 \varepsilon / \sigma^{2}$.

We include a bond-bending potential [14-16] that increases the rigidity of the chain:

$V_{B}(r)=b \sum_{i=2}^{N-1}\left(1-\frac{\left(\vec{r}_{i-1}-\vec{r}_{i}\right) \cdot\left(\vec{r}_{i}-\vec{r}_{i+1}\right)}{\left|\left(\vec{r}_{i-1}-\vec{r}_{i}\right) \cdot\left(\vec{r}_{i}-\vec{r}_{i+1}\right)\right|}\right)$

where $\vec{r}_{i}$ denotes the position of the bead $\mathrm{i}$ along the chain and $\mathrm{b}$ characterizes the stiffness. In this work, the equations of motion are solved using the velocity Verlet algorithm with a 
time step of $\mathrm{dt}=0.001 \tau_{\mathrm{LJ}}$. The temperature of the system was controlled with a Nose-Hoover thermostat (LAMMPS) [17].

The present model is composed of 320 linear polymer chains with $\mathrm{N}=100$ or $\mathrm{N}=200$ spherical beads of masse $\mathrm{m}$. The polymer chains are placed in a cubic simulation box with periodic boundary conditions in the three directions. The initial configuration of the polymer chain is a random walk at a low initial density $(\mathrm{d} \approx 0.8)$. The successive beads are separated by a distance equal to the equilibrium bond length, $0.96 \sigma$. The minimum distance between next-nearest neighbours is set to $1.02 \sigma$. In this initial configuration, many monomers may overlap. In order to equilibrate the chains, we use standard molecular dynamics equilibration methods [14-16]. To gradually remove the overlap between the chains, a NVT simulation is done with a repulsive cosine potential for non bonded interactions [14-16]. The magnitude of the cosine potential is gradually increased over the run. Then, this soft potential is replaced by a standard Lennard-Jones repulsion between the monomers. The full Van der Waals potential is then introduced progressively with increasing values of $\sigma$. The system is equilibrated further for one million steps using the full potential and standard NPT simulations at T=1.0 and with a compressive pressure $\mathrm{P}=1.0$. As a glassy is desired, the system is then rapidly cooled to $\mathrm{T}=0.1<\mathrm{T}_{\mathrm{g}}$ at a cooling rate $d T / d t=-210^{-3}(\varepsilon / k) / \tau_{L J}$ and relaxed for 1 million steps further with a small compression. This equilibration procedure produced an equilibrated state from which we could begin the simulations. In the following, we have studied the effect of the chain rigidity. We have considered two cases, with the stiffness parameters $b=0$ and $b=2$ corresponding to a flexible and a semi-flexible chain respectively. We have also investigated the effect of the Van der Waals interactions. The Lennard Jones potential cutoff distance is set to $r_{c}=1.65$ or $r_{c}=2.2$ corresponding to weak and strong intermolecular interactions respectively [14-16]. For the semi-flexible chain $(b=2)$, runs with $r_{c}=2.2$ and $r_{c}=1.65$ were performed at the initial density $\rho=1.05$ and $\rho=1.00$ respectively. In the runs with $b=0$ and $r_{c}=1.65$, the resulting 
glasses have the starting density $\rho=1.02$. The radius of gyration of the polymer chains scales as $\mathrm{R}_{\mathrm{g}}{ }^{2}=0.28(\mathrm{~N}-1) \sigma^{2}$ and $\mathrm{R}_{\mathrm{g}}{ }^{2}=0.54(\mathrm{~N}-1) \sigma^{2}$ for the flexible and semi-flexible chain respectively in agreement with $[2,15]$. For the bead-spring models like the ones studied in this work, the entanglement lengths $\mathrm{N}_{\mathrm{e}}$ vary from 70 for fully flexible chains to 20 for semi-flexible chains with $b=2[1,16,18-19]$. The chains investigated here are long enough to entangle, i.e., $N>N_{e}$. Robbins et al. demonstrated the importance of chain length for the onset of craze growth. Yet, in our work, similar results were obtained for $\mathrm{N}=100$ and $\mathrm{N}=200$ suggesting that the chain length has no role for the first steps of the cavitation process as long as the cavity size remains small compared to both $\mathrm{N}$ and $\mathrm{N}_{\mathrm{e}}$. Only the results corresponding to $\mathrm{N}=100$ are detailed in the following.

In this work, the temperature is maintained constant at $\mathrm{T}=0.1$, well below the glass transition temperature which lies in the range $0.3-0.5$ for the systems under study [14-16,18-19]. Starting from the relaxed configurations, an hydrostatic deformation is then applied on the three axes of the simulation cell at a constant strain rate of $\dot{\varepsilon}_{X}=\dot{\varepsilon}_{Y}=\dot{\varepsilon}_{Z}=510^{-3} / \tau_{L J}$. The results presented in the following are qualitatively the same in the strain rate range $2.10^{-3}$ $5.10^{-3} / \tau_{\mathrm{LJ}}$. The measured stress used in this work is the averaged hydrostatic virial stress $\sigma=\left(\sigma_{x x}+\sigma_{y y}+\sigma_{z z}\right) / 3$. For each value of the cutoff $r_{c}$ and of the stiffness $b$, three separate cells were built to ensure that a range of conformations were explored. In order to improve the statistics of the results, an average was taken over the results obtained with the three structures. 


\section{Results and discussion}

The simulated stress-strain responses obtained display the features observed experimentally and in similar coarse grain simulations [14-16]. Figure 1 shows the evolution at $\mathrm{T}=0.1$ of the hydrostatic stress $\sigma$ as a function of the relative volume variation $\Delta \mathrm{V} / \mathrm{V}$ for weak and strong interactions and for the rigid and flexible chains. Initially, a linear elastic response is obtained. A stress peak is then observed and it is followed by a softening of the stress-strain response. For the semi-flexible structures $(b=2)$, the model with a high interaction strength $\left(r_{c}=2.2\right)$ has a higher modulus. The system with the highest modulus is the one with $r_{c}=1.65$ and $b=0$, in agreement with the results of B.Schnell et al.[2]. The strength of the intermolecular potential is the main parameter determining the modulus. Yet, the equilibrium chain angles distribution and the chain conformations are modified in the semi-flexible chain model so that the small chain rearrangements through rotations are easier for small deformations in these structures than in the flexible ones [2]. In the following, we will analyze successively the free volume increase and the mobility heterogeneities associated to the cavitation process and the role played by the chain ends.

\section{$\underline{\text { Hard probe free volume analysis and Voronoï tessellation }}$}

The free volume concept has been often used to explain the behaviour of glassy materials. For the evaluation of the free volume distribution, several approaches have been followed, the hard spherical probe method [20, 21] and the Voronoï tessellation method [22]. First, we have studied in detail the free volume size and distribution especially at the stress peak with the hard spherical probe method detailed by Mattice et al $[20,21]$. To evaluate the cavities, the periodic box is divided into cubic subcells of size $\Delta$ and the atoms are assumed to be hard spheres with a diameter equal to $\sigma$. In order to check occupancy, a probe of diameter $\sigma$ is introduced in the simulation cell. Each atom is assigned an effective radius which the sum of 
its hard core radius and the probe radius. Subcells whose center is within the effective radius of any atom are assumed to be occupied. The subcell is labelled as unoccupied when it is not recovered by the hard sphere. The cavities were probed throughout the structure and the unoccupied cells were counted as holes contributing to the free volume. Two unoccupied subcells are assigned to the same hole when they share a common face. Figure 2 displays the evolution of the free volume fraction when the grid size $\Delta$ is varied between $0.5 \sigma$ and $2 \sigma$ for the semi-flexible chain with weak intermolecular interactions $\left(r_{c}=1.65\right)$. With the cell size $\Delta=2 \sigma$, almost no free volume is available below the stress peak while with $\Delta=0.5 \sigma$ a significant amount of free volume is present in the cell at the beginning of the run. In the following, we have chosen the probe size $\Delta=\sigma$. With this choice, the free volume fraction increases drastically at the stress peak. This free volume is related to the formation of small cavities of size larger than $\sigma$. In the following, we focus on the free volume size, shape and distribution at the stress peak. The cavities constantly change their shape and size. In an attempt to obtain results that are representative, five additional snapshots are selected before and after the stress peak corresponding to $\Delta \mathrm{V} / \mathrm{V}<0.01$ around the peak and an average is performed for the volume size and distribution over the ten structures considered and over the three starting cells, for a given value of $b$ and $r_{c}$. Figures 3.a and 3.b and Table 1 compare the hole size distributions obtained for weak $\left(r_{c}=1.65\right)$ and strong $\left(r_{c}=2.2\right)$ interactions and flexible $(b=0)$ and semi-flexible chains $(b=2)$. Numerous small holes are obtained for weak intermolecular interactions $\left(r_{c}=1.65\right)$ and the cavities sizes distribution is very broad. When the intermolecular interactions are weak, the cavitation regions are very diffuse and the localization is weak. On the contrary, for strong interactions, larger holes are obtained, with a very narrow distribution. In the case of the flexible chain, medium size cavities are found. The hole size distribution width is intermediate between the two former ones. These findings indicate a relationship between the first stages of the cavitation process and the strength of the 
intermolecular interactions and the flexibility of the chain. The localization of the process is enhanced for strong intermolecular interactions and a flexible chain.

Following Mattice et al. [20, 21], the shape and symmetry of the cavities at the stress peak has been evaluated through their asphericity and acylindricity. The symmetry was analysed using the principal moments of the radius of gyration tensor of the voids. The three eigenvalues were obtained in the order $R_{1}^{2}>R_{2}^{2}>R_{3}^{2}$ for each void. The asphericity, $\sigma$, can be calculated by using:

$\sigma=\frac{R_{1}{ }^{2}-\frac{R_{2}{ }^{2}+R_{3}{ }^{2}}{2}}{R_{g}{ }^{2}}$

and the acylindricity $\chi$ can be measured by:

$\chi=\frac{R_{2}{ }^{2}-R_{3}{ }^{2}}{R_{g}{ }^{2}}$

where $\mathrm{R}_{\mathrm{g}}{ }^{2}$ is the squared radius of gyration, which is the sum of the $\mathrm{R}_{\mathrm{i}}{ }^{2}$.

The upper limits of asphericity and acylindricity defined here are 1 and $1 / 2$ respectively. The lower limits are zero and are a obtained with the maximum corresponding symmetry [20, 21]. The averaging procedure is the same as the one used for the free volume analysis: ten additional snapshots are selected corresponding to $\Delta \mathrm{V} / \mathrm{V}<0.01$ around the stress peak and an average is performed for the asphericity and acylindricity over the ten snapshots and over the three starting structures. The average values of the asphericity and acylindricity obtained are summarized in Table 1. Our results suggest a higher spherical symmetry for strong intermolecular interactions $\left(r_{c}=2.2\right)$. The hole shape departs from spherical symmetry and is more cylindrical for weak intermolecular interactions $\left(r_{c}=1.65\right)$. The spherical symmetry obtained for the flexible chain $(b=0)$ is slightly lower than the one found for strong intermolecular interactions. 
To conclude, our findings suggest that the balance between the intramolecular and intermolecular terms of the potential determines the holes distribution and shape. A more localized cavitation process and a spherically isotropic free volume topology is found for strong intermolecular interactions or very flexible chains. On the contrary, weaker intermolecular interactions lead to a more diffuse cavitation process, with a more cylindrical shape of the cavities. On the one hand, as pointed by Stachurski [22], the molecular properties and the free volume increase in amorphous polymers must not be thought as spherically isotropic, as in liquid and gases, since the free volume expansion is derived from a two dimensional motion orthogonal to the chain axis. According to our results, this anisotropy is enhanced for strong intramolecular interactions. On the other hand, it has already been suggested that different deformation mechanisms are involved in amorphous polymers depending on the chain rigidity and on the intermolecular interactions. In the framework of the "strophon" model of Yannas et al. [22, 23], a deforming polymer can undergo cavitation or shear yielding depending on the relative strength of the intermolecular interactions and of the conformational barriers. When the intramolecular forces exceed the intermolecular forces, yielding is expected to be the prevailing mechanism. On the contrary, if the intermolecular potential is strong, crazing and cavitation is the dominant deformation process. The fact that a diffuse cavitation process is obtained in our work for weak intermolecular interactions $\left(r_{c}=.65\right)$ may indicate that some plastic flow enters in competition with the cavitation phenomenon in these structures, while this deformation mechanism was negligible for strong intermolecular interactions $\left(r_{c}=2.2\right)$. As already mentioned, small angles changes are possible in the semi-flexible structure $(b=2)[2]$ that may lead to a plastic activity at the expense of the cavitation process. These small rearrangements are not allowed in the flexible structure. A strong and localized cavitation process is thus the dominant deformation mechanism in the flexible structure $(b=0)$ at the expense of shear plasticity. 
In order to complement the hard probe free volume analysis, we have determined the available space surrounding a bead and the evolution of the free volume with a Voronoï tessellation [24]. In the Voronoi polyhedra analysis, one perperpendicularly bisects the segments connecting a chosen bead and its nearest neighbours. The Voronoi polyhedron is then obtained by selecting the smallest of the polyhedra formed by the intersecting planes. Every point within the Voronoï polyhedron is closer to the chosen bead than to any other atom in the system. The simulation cell is thus divided into convex polyhedra surrounding each atom. In order to characterize the structure of the system, we have calculated the evolution of the mean Voronoï polyhedra volume per bead, $\left\langle\mathrm{V}_{\mathrm{p}}\right\rangle$, with the hydrostatic deformation. As illustrated in Figure 4, for strong intermolecular interactions or a weak angular potential, low values of the Voronoï volumes are found, which is in agreement with the densities obtained for the equilibrated structures. The mean Voronoï polyhedra volume shifts to larger values during the first stages of the hydrostatic deformation. After the stress peaks, which are indicated with black arrows on the Figure 4, the increase is less marked and there is no significant change in the local packing of the chains. This result suggests that before the stress peak, the increase of the free volume is dominated by an homogeneous bead separation and local rearrangements, even if small cavities are formed. After the stress peaks, the free volume increase is associated to the growth of the existing cavities.

The evolution with deformation of the standard deviations of the Voronoi volume distributions are displayed in Figure 5. This standard deviation is higher for weak intermolecular interactions than for strong interactions or for the flexible chain. This result may be attributed to an increasing local disorder and free volume heterogeneity inside the polymer for decreasing Van der Waals interactions. The Voronoï tessellation analysis also shows a broadening of the distribution of polyhedra volumes during the first stages of the expansion of the system. As the volume is further increased, the distribution width becomes 
constant. The free volume distribution and local packing disorder increases until the stress peak but the growth of the cavities after this peak does not affect much the free volume heterogeneity.

\section{Chain ends effects}

The chain length dependence of the glass transition temperature of polymers has often been explained by the larger free volume surrounding a chain end [25]. In the non deformed structures, the Voronoï polyhedron volume associated to the chain ends beads is a few percent higher than the average Voronoï volume. Yet, no clear tendency as a function of the chain stiffness or as a function of the strength of the interactions could be evidenced. These findings are in agreement with the results reported by Rigby at al. [24]. The chain end may thus act like a defect which favour the cavitation process. In order to study the correlations between the chain ends and the holes at the stress peak, we have calculated a radial distribution function $g_{A B}(r)$ where A corresponds to a chain end and B to an elementary cell of size $\sigma^{3}$ inside a cavity. This function gives the probability of finding a pair A-B with a separation of $r$, relative to the probability expected for a uniform distribution of the A-B pairs:

$$
g_{A B}(r)=\frac{n_{A B}(r)}{4 \pi r^{2} \Delta r \rho_{A B}}
$$

In this formula, $\mathrm{n}_{\mathrm{AB}}$ is the average number of $\mathrm{AB}$ pairs in the spherical shell between $\mathrm{r}$ and $r+\Delta r$, and $\rho_{A B}$ is the density of $A B$ pairs. The radial distribution functions obtained at the stress peak are displayed in Figure 6. Ten configurations were selected around the stress peak and the same averaging procedure as before was applied. When the strength of the intermolecular interactions increases, the maximum of $\mathrm{g}_{\mathrm{AB}}(\mathrm{r})$ and the whole distance distribution is shifted to lower values and the peak magnitude increases. A similar result is obtained when the flexibility of the chain is increased. The spatial correlations between 
between holes and end chains are thus higher for strong intermolecular interactions and for the flexible chain.

\section{$\underline{\text { Mobility heterogeneities }}$}

Several molecular dynamics simulations have suggested that the deformation process is associated in amorphous polymers with stress, deformation and mobility heterogeneities [4, 5]. On the other hand, in the framework of the free volume theories, every type of molecular motion needs its own free volume and the molecular mobility is dependent on the probability that a molecule is next a hole of size sufficient for displacement [25]. The hydrostatic deformation is likely to induce an additional volume in the polymer that causes an increase in mobility heterogeneities.

In order to characterize the mobility heterogeneities, we have calculated the evolution of fourth order centered moment of the velocity distributions as a function of the relative variation of the simulation cell volume $\Delta \mathrm{V} / \mathrm{V}$. This moment is defined as:

$$
m_{4}=\frac{<(v-<v>)^{4}>}{<v>^{4}}
$$

where $\mathrm{v}$ is the velocity and $\langle\mathrm{v}>$ its average value. This quantity reveals the large deviations from the average value of the velocity. Figure 7 displays the evolution of the fourth order moment of the velocity distribution in the flexible and semi-flexible case and for weak and strong intermolecular interactions as a function of the relative volume variation $\Delta \mathrm{V} / \mathrm{V}$. On this figure, the stress peaks are indicated with black arrows. This figure illustrates the increase of the mobility heterogeneity with the increase of the volume of the simulation cell upon hydrostatic deformation. Large velocity fluctuations are observed before the stress peak and they are enhanced at the stress peak. A limited number of regions have thus a high molecular mobility. This result is in agreement with the stress induced increase of the torsional mobility reported by Capaldi et al. [3] before and just beyond the yield point. The cavitation process may thus be related to the mobility heterogeneities. A possible interpretation is that the atoms 
with a high mobility deform more easily and that the highly mobile regions act as nucleation sites for the cavitation process. As shown on Figure 7, the mobility fluctuations in the early stages of the cavitation process are affected by the strength of the intermolecular interactions and by the chain flexibility. These velocity fluctuations are enhanced when the strength of the intermolecular interactions increases. Moreover, the decrease of the chain rigidity leads to large mobility heterogeneities. This result confirms the fact that a higher localization of the cavitation process is obtained for strong intermolecular interactions or for the flexible chain, as illustrated in the free volume study.

In order to study in more details the relationships between mobility heterogeneities and cavitation, we have calculated the velocity probability distributions tails $\mathrm{P}(\mathrm{v}>10<\mathrm{v}>)$, $\mathrm{P}(\mathrm{v}>5<\mathrm{v}>), \mathrm{P}(\mathrm{v}<0.1<\mathrm{v}>), \mathrm{P}(\mathrm{v}<0.2<\mathrm{v}>)$, which represent the probability $\mathrm{P}$ that the velocity $\mathrm{v}$ is higher or lower than a certain fraction $(10,5,0.1,0.2)$ of the average velocity value, $<v>$. Figure 8 displays the evolution of these probability distributions tails as a function of the volume relative variation $\Delta \mathrm{V} / \mathrm{V}$ for the semi-flexible chain with weak intermolecular interactions $\left(b=2, r_{c}=1.65\right)$. The values are normalized by the values obtained in the non deformed structure for $\Delta \mathrm{V} / \mathrm{V}=0$, denoted as $\mathrm{P}_{\text {ini. }}$. The probabilities distribution tails $\mathrm{P}(\mathrm{v}>5<\mathrm{v}>)$ and $\mathrm{P}(\mathrm{v}<0.2<\mathrm{v}>)$ depart from their initial values before the stress peak. The velocity distributions are thus shifted towards low and high values during the first stages of the cavitation process. The larger and lower velocities corresponding to $\mathrm{P}(\mathrm{v}>10<\mathrm{v}>)$ and $\mathrm{P}(\mathrm{v}<0.1<\mathrm{v}>)$ appear at the stress peak. The same qualitative findings are obtained for strong interactions and for the flexible chains, with enhanced velocity fluctuations.

In an attempt to compare the velocity fluctuations in the whole simulation cell and in the vicinity of the holes, we have calculated the standard deviation of the velocity distribution, $\left.\mathrm{m}_{2}=<(\mathrm{v}-\langle\mathrm{v}\rangle)^{2}\right\rangle /\langle\mathrm{v}\rangle^{2}$, when only atoms at a distance lower than $3 \sigma$ from a cavity with a size larger than $\sigma^{3}$ are taken into account. In view of the limited number of atoms considered, the 
fourth order moment is in this case a too noisy quantity. Figure 9 displays the evolution of the standard deviation as a function of the volume relative variation $\Delta \mathrm{V} / \mathrm{V}$ for strong intermolecular interactions $\left(r_{c}=2.2\right)$ in the vicinity of the cavities and in the whole simulation box. In the limit of no deformation, no holes are found and the velocity fluctuations near the holes are therefore equal to zero. For increasing deformation, the holes appear and the velocity heterogeneities are more marked in the vicinity of the holes. A similar result was obtained for weaker interactions and for the flexible chain. A possible explanation is that the cavities created during the hydrostatic deformation are disordered regions, and that local rearrangements are easier near these free volume regions, generating large velocity fluctuations.

\section{Conclusion}

In this work, we have investigated with coarse grain molecular dynamics simulations the first stages of the cavitation process in an amorphous polymer below $\mathrm{T}_{\mathrm{g}}$ upon hydrostatic deformation. The free volume size and shape is affected by the chain flexibility and by the strength of the intermolecular interactions. For strong intermolecular interactions or a flexible chain, the cavitation process is highly localized and the holes have a high spherical symmetry. The cavitation regions are more diffuse for weaker intermolecular interactions. The mean Voronoï polyhedra volume and the free volume heterogeneity, as determined from a Voronoï tessellation, increase until the glassy stress peak. After this peak, the increase of the free volume can be attributed to the growth of the existing cavities. The holes nucleate preferentially near the chain ends of the polymer. High mobility regions are present before the stress peak. These high mobility regions may enable the nucleation of cavities in the bulk. The 
localization of these high mobility zones is higher for strong intermolecular interactions. Moreover, the velocity fluctuations are markedly enhanced in the vicinity of the holes. Although many questions about the molecular origin of cavitation in polymers remain unanswered, it is shown that molecular simulations can be a valuable tool to capture some information on the localization of the cavitation process and on the relationships between the mobility heterogeneities and the first stages of this phenomenon. 


\section{References}

[1] J.Rottler,M.O.Robbins. Growth, microstrucutre and failure of crazes in glassy polymers. Physical Review E 68, 011801 (2003).

[2] B.Schnell. Etude par simulation numérique de la transition vitreuse et de l'état vitreux de polymers denses amorphes: propriétés mécaniques et phénomènes de cavitation. PHD Thesis Louis Pasteur University (2006).

[3] F.C.Capaldi, M.B.Boyce, G.C.Rutledge. Enhanced mobility accompanies the active deformation of a glassy amorphous polymer. Physical Review Letters Vol.89, 17, 175505 (2002).

[4] W.Kob, C.Donati,S.J.Plimpton, P.H.Poole, S.C.Glotzer. Dynamical heterogeneities in supercooled Lennard-Jones liquid. Physical Review Letters Vol 79, N 15,2827 , (1997).

[5] S.C.Glotzer, C.Donati. Quantifying spatially heterogenous dynamics in computer simulations of glass-forming liquids. J.Phys.Condens.Matter. 11, A285-295, (1999).

[6] T.Muranaka, Y.Hiwatari. Structural heterogeneity in supercooled liquid and glass. Journal of Non Crystalline Solids 19, 235-237, (1998).

[7]M.D.Ediger. Spatially heterogeneous dynamics in supercooled liquids. Annu.Rev.Phys.Chem. 51, 99, (2000).

[8] R.Richert. Heterogeneous dynamics in liquids: fluctuations in space and time. J.Phys.:Condens.Matter, 14 R, 703, (2002).

[9] Y.Jin , R.H.Boyd. Subglass chain dynamics and relaxation in polyethylene: a molecular dynamics simulation study. J.Chem.Phys. 108, 9912, (1998).

[10] S.Shenogin, R.Ozisik. Simulation of plastic deformation in glassy polymers: atomistic and mesoscale approaches. Journal of Polymer Science Part B: Polymer Physics 43, 994, (2005). 
[11] S.Shenogin, R.Ozisik. Deformation of glassy polycarbonate and polystyrene: the influence of chemical structure and local environment. Polymer 46, 4397, (2005).

[12] K.Yoschimoto, T.Jain, K.V.W.Workum, P.F.Neally, J.J. de Pablo. Mechanical heterogeneities in model polymer glasses at small length scales Physical Review Letters 93, 175501, (2004).

[13] W.Paul, G.D.Smith.Structure and dynamics of amorphous polymers: computer simulations compared to experiment and theory. Rep.Prog.Phys. 1117, 67, (2004).

[14] A.R.C.Baljon, M.O.Robbins. Simulations of crazing in polymer glasses: effect of chain length and surface tension. Macromolecules 34, 4200, (2001).

[15] K.Kremer, G.S.Grest. Dynamics of entangled linear polymer melts: a molecular dynamics simulation. J.Chem.Phys. 92, 5057, (1990).

[16] M.Pütz, K.Kremer, G.S.Grest. What is the entanglement length in a polymer melt? Europhys.Lett. 735, 49, (2000).

[17] S.Nose. A unified formulation of the constant temperature molecular dynamics methods. J.Chem.Phys. 81, 511 (1984).

[18] R.Everaers, S.K.Sukumaran, G.S.Grest, C.Svaneborg, A.Sivasubramanian, K.Kremer. Rheology and microscopic topology of entangled polymeric liquids. Science 303, 823 (2004). [19] Q.Zhou, R.G.Larson. Primitive path identification and statistics in molecular dynamics simulations of entangled polymer melts. Macromolecules 38, 5761, (2005).

[20] W.K.Kim, W.L.Mattice. A fully atomistic model of an amorphous polybenzoxazine at bulk density. Computational and Theoretical Polymer Science Vol.8, N³/4, 353-361 (1998)

[21] S.Misra, W.L.Mattice. Atomistic models of amorphous polybutadienes. Static free volume. Macromolecules 26, 7274, (1993).

[22] Z.H.Stachurski, Deformation mechanisms and yield strength in amorphous polymers Prog.Polym.Sci., Vol. 22, 407, (1997). 
[23] I.V.Yannas, R.R.Luise The strophon theory of deformation in glassy polymers: application to small deformation, in the strength and stiffness of polymers Zachariades, A.E R.S.Porter (Eds), New York: Marcel Dekker. (1983)

[24] D.Rigby, R.J.Roe. Molecular dynamics simulation of polymer liquid and glass. A free volume distribution. Macromolecules 23, 5312, (1990).

[25] T.G.Fox, P.J.J.Flory. Second-order transition temperatures and related properties of polystyrene I. Influence of molecular weight. J.Appl.Phys. 21, 581, (1950).

[26] A.Doolittle. Studies in Newtonian Flow II. The dependence of the viscosity of liquids on free space. J.Appl.Phys.22, 1471, (1951). 


\section{Figure caption}

Figure 1: Evolution of the hydrostatic stress $\sigma$ as a function of the relative variation of the volume $\Delta \mathrm{V} / \mathrm{V}\left(\boldsymbol{\square} \mathrm{r}_{\mathrm{c}}=2.2, \mathrm{~b}=2, \square \mathrm{r}_{\mathrm{c}}=1,65, \mathrm{~b}=0, \mathrm{O} \mathrm{r}_{\mathrm{c}}=1,65, \mathrm{~b}=2\right)$.

Figure 2: Evolution of the hydrostatic stress $\sigma(\bullet)$, of the free volume fraction for the grid size values $\Delta=0.5 \sigma(\boldsymbol{\square}), \Delta=1 \sigma(O)$, and $\Delta=2 \sigma(+)$ for the semi-flexible (b=2) chain with weak intermolecular interactions $\left(\mathrm{r}_{\mathrm{c}}=1.65\right)$.

Figure 3.a: Hole size distributions obtained at the stress peak for weak ( $\left.\square r_{c}=1.65, b=2\right)$ and strong $\left(\boldsymbol{\square} \mathrm{r}_{\mathrm{c}}=2.2, \mathrm{~b}=2\right)$ interactions.

Figure 3.b: Hole size distributions obtained at the stress peak for flexible $\left(\bullet r_{c}=1.65, b=0\right)$ and semi-flexible chains $\left(\square r_{c}=1.65, b=2\right)$.

Figure 4: Evolution of the average Voronoï polyhedra volume, $\left\langle\mathrm{V}_{\mathrm{p}}\right\rangle$, as a function of the simulation cell relative volume variation $\Delta \mathrm{V} / \mathrm{V}\left(\boldsymbol{\nabla} \mathrm{r}_{\mathrm{c}}=2.2, \mathrm{~b}=2, \bullet \mathrm{r}_{\mathrm{c}}=1.65, \mathrm{~b}=0, \bigcirc \mathrm{r}_{\mathrm{c}}=1.65\right.$, $b=2$ ). The stress peak position is indicated with a black arrow.

Figure 5: Evolution of the standard deviation of the Voronoï polyhedra volume $\mathrm{V}_{\mathrm{p}}$ as a function of the simulation cell relative volume variation $\Delta \mathrm{V} / \mathrm{V}\left(\boldsymbol{\square} \mathrm{r}_{\mathrm{c}}=2.2, \mathrm{~b}=2, \bullet \mathrm{r}_{\mathrm{c}}=1.65\right.$, $\left.b=0, \circ r_{c}=1.65, b=2\right)$. The stress peak position is indicated with a black arrow.

Figure 6: Radial distribution function $\mathrm{g}_{\mathrm{AB}}(\mathrm{r})$, with $\mathrm{A}=$ chain end and $\mathrm{B}=$ elementary cell of size $\sigma^{3}$ inside a cavity, $\left(\square r_{c}=2.2, b=2\right),\left(\square r_{c}=1,65, b=0\right),\left(\bullet r_{c}=1.65, b=2\right)$. 
Figure 7: Evolution of the fourth order moment of the velocity distribution, $\mathrm{m}_{4}$, as a function of the relative variation of the volume $\Delta \mathrm{V} / \mathrm{V}\left(\square \mathrm{r}_{\mathrm{c}}=2.2, \mathrm{~b}=2, \square \mathrm{r}_{\mathrm{c}}=1.65, \mathrm{~b}=0, \bigcirc \mathrm{r}_{\mathrm{c}}=1.65\right.$, $b=2$ ). The stress peak position is indicated with a black arrow.

Figure 8.a: Velocity probability distributions tails $\mathrm{P}(\mathrm{v}<0.1<\mathrm{v}>)$ as a function of the relative variation of the volume $\Delta \mathrm{V} / \mathrm{V}$ for the semi-flexible chain with weak intermolecular interactions $\left(b=2, r_{c}=1.65\right)$.

Figure 8.b: Velocity probability distributions tails $\mathrm{P}(\mathrm{v}<0.2<\mathrm{v}>)$ as a function of the relative variation of the volume $\Delta \mathrm{V} / \mathrm{V}$ for the semi-flexible chain with weak intermolecular interactions $\left(b=2, r_{c}=1.65\right)$.

Figure 8.c: Velocity probability distributions tails $\mathrm{P}(\mathrm{v}>5<\mathrm{v}>)$ as a function of the relative variation of the volume $\Delta \mathrm{V} / \mathrm{V}$ for the semi-flexible chain with weak intermolecular interactions $\left(b=2, r_{c}=1.65\right)$.

Figure 8.d: Velocity probability distributions tails $\mathrm{P}(\mathrm{v}>10<\mathrm{v}>)$ as a function of the relative variation of the volume $\Delta \mathrm{V} / \mathrm{V}$ for the semi-flexible chain with weak intermolecular interactions $\left(b=2, r_{c}=1.65\right)$.

Figure 9: Standard deviation of the velocity distribution, $\mathrm{m}_{2}$, in the whole simulation box $(\boldsymbol{\square})$ and in the vicinity of the holes $(\square)$, when the bead-hole distance is lower than $3 \sigma$, for $r_{c}=1.65$ and $b=2$. The stress peak position is indicated with a black arrow. 


\section{Table 1}

Average hole size $\left(\sigma^{3}\right)$, standard deviation of the hole size, asphericity, $\sigma$, and acylindricity, $\chi$, evaluated at the stress peak.

\begin{tabular}{|c|c|c|c|}
\hline & $\mathrm{b}=0 \mathrm{r}_{\mathrm{c}}=1.65$ & $\mathrm{~b}=2 \mathrm{r}_{\mathrm{c}}=1.65$ & $\mathrm{~b}=2 \mathrm{r}_{\mathrm{c}}=2.2$ \\
\hline $\begin{array}{c}\text { Average hole size } \\
\left(\sigma^{3}\right)\end{array}$ & 5.8 & 4.3 & 8.5 \\
\hline $\begin{array}{c}\text { Standard deviation of } \\
\text { the hole size }\end{array}$ & 0.8 & 1.35 & 0.28 \\
\hline$\sigma$ & 0.35 & & 0.30 \\
\hline$\chi$ & 0.10 & 0.50 & 0.15 \\
\hline
\end{tabular}




\section{Page 23 of 35}

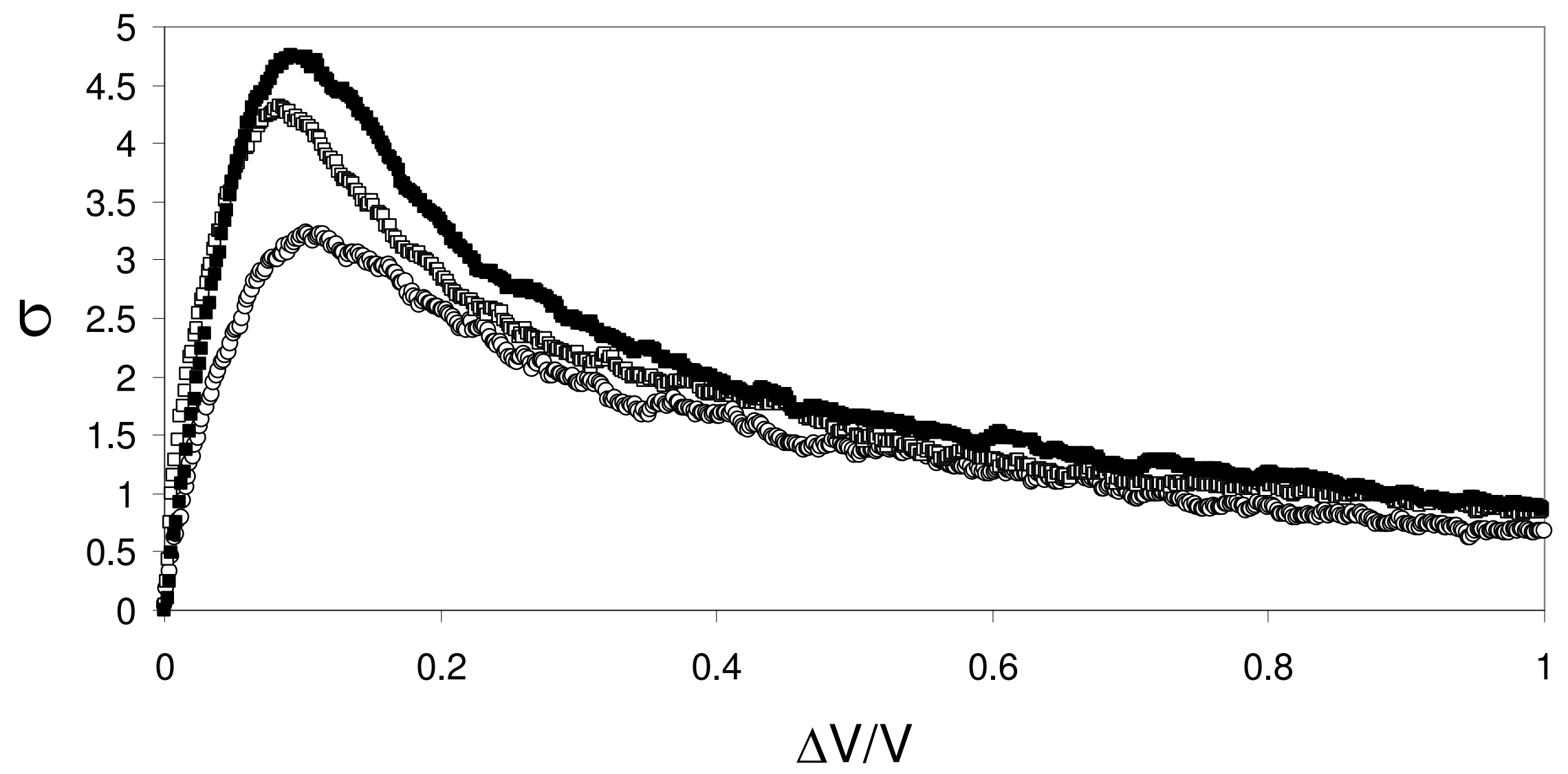

http://mc.manuscriptcentral.com/tandf/jenmol 


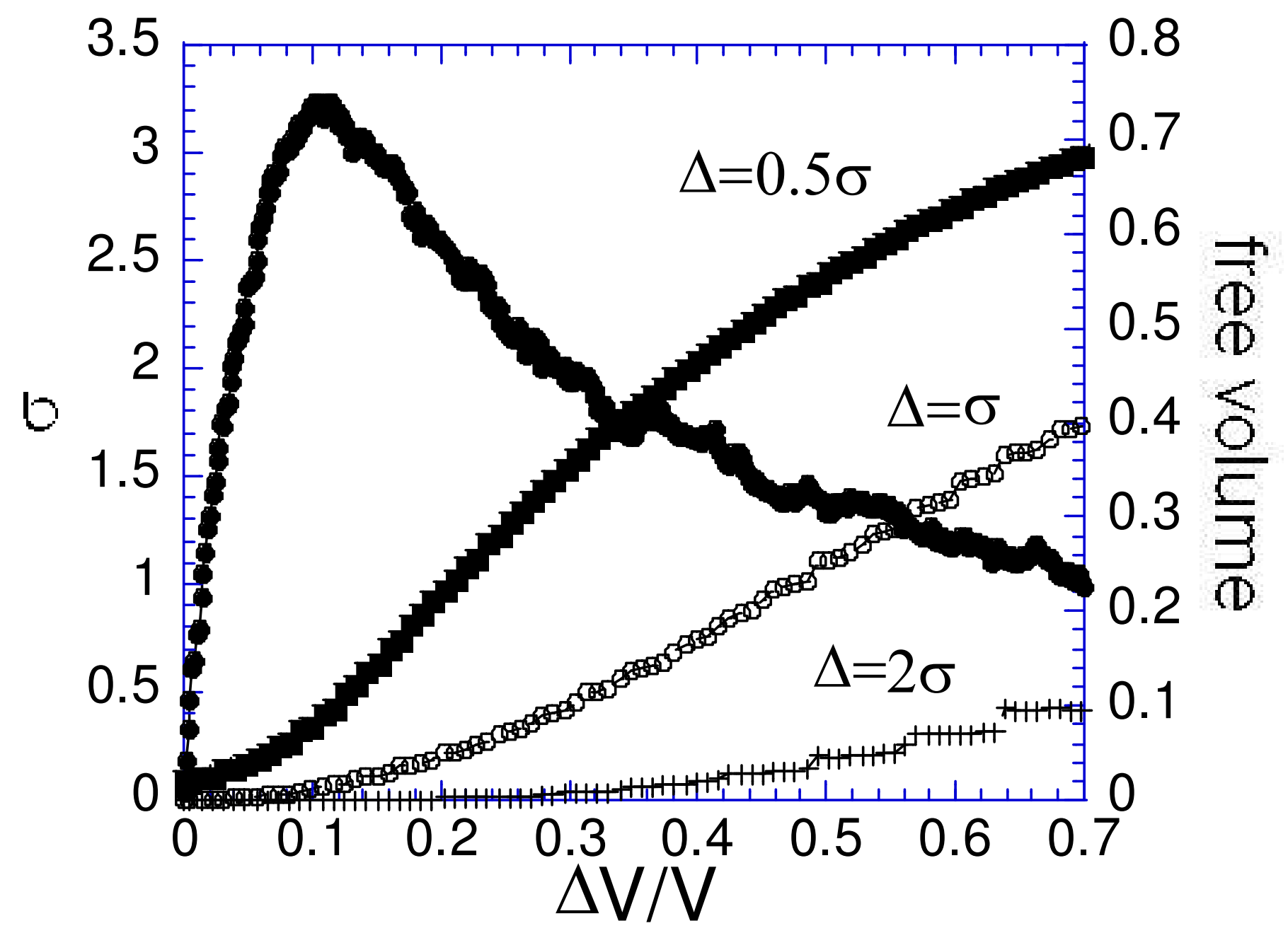

http://mc.manuscriptcentral.com/tandf/jenmol 


\section{Page 25 of 35}

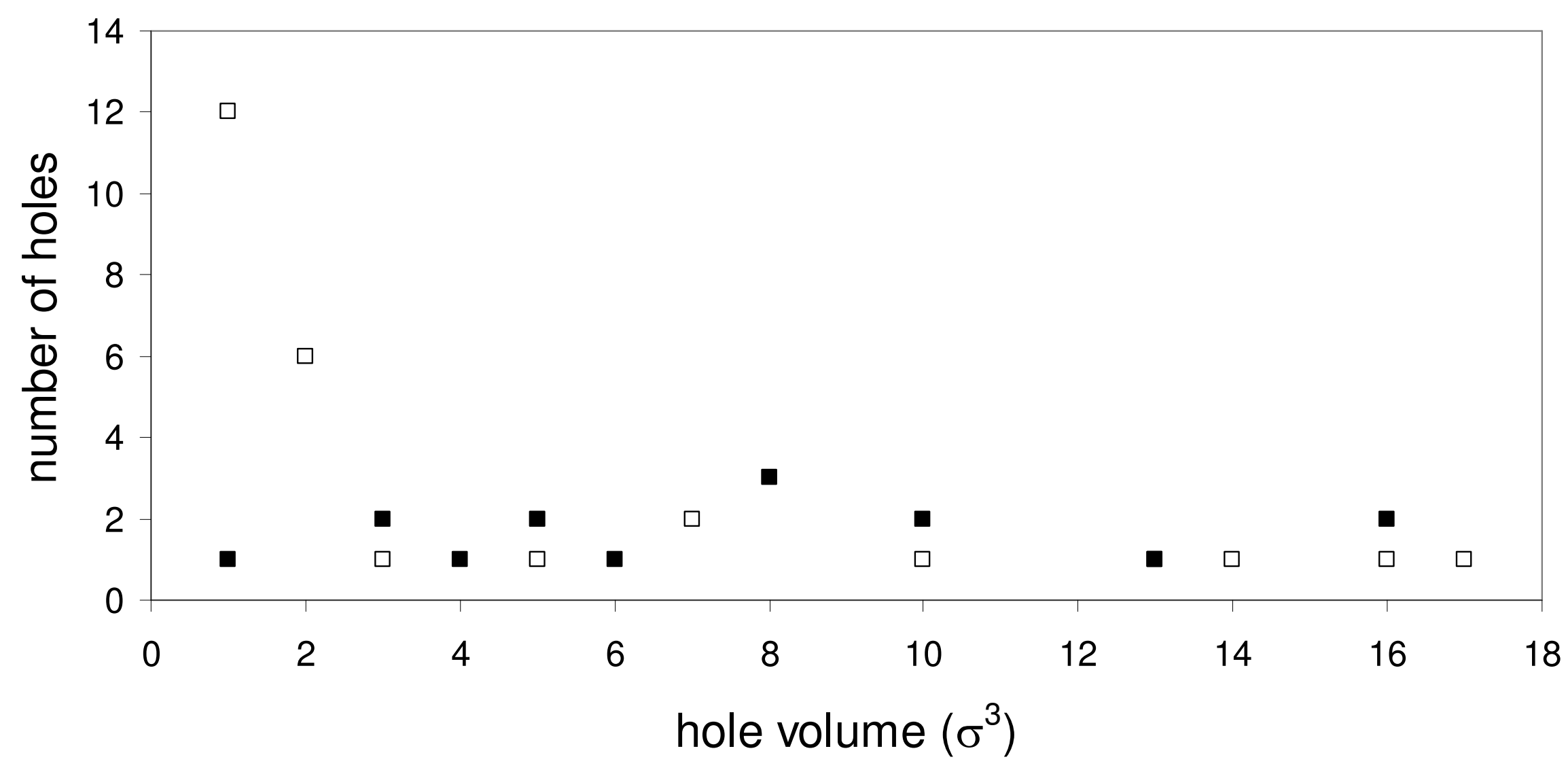

http://mc.manuscriptcentral.com/tandf/jenmol 


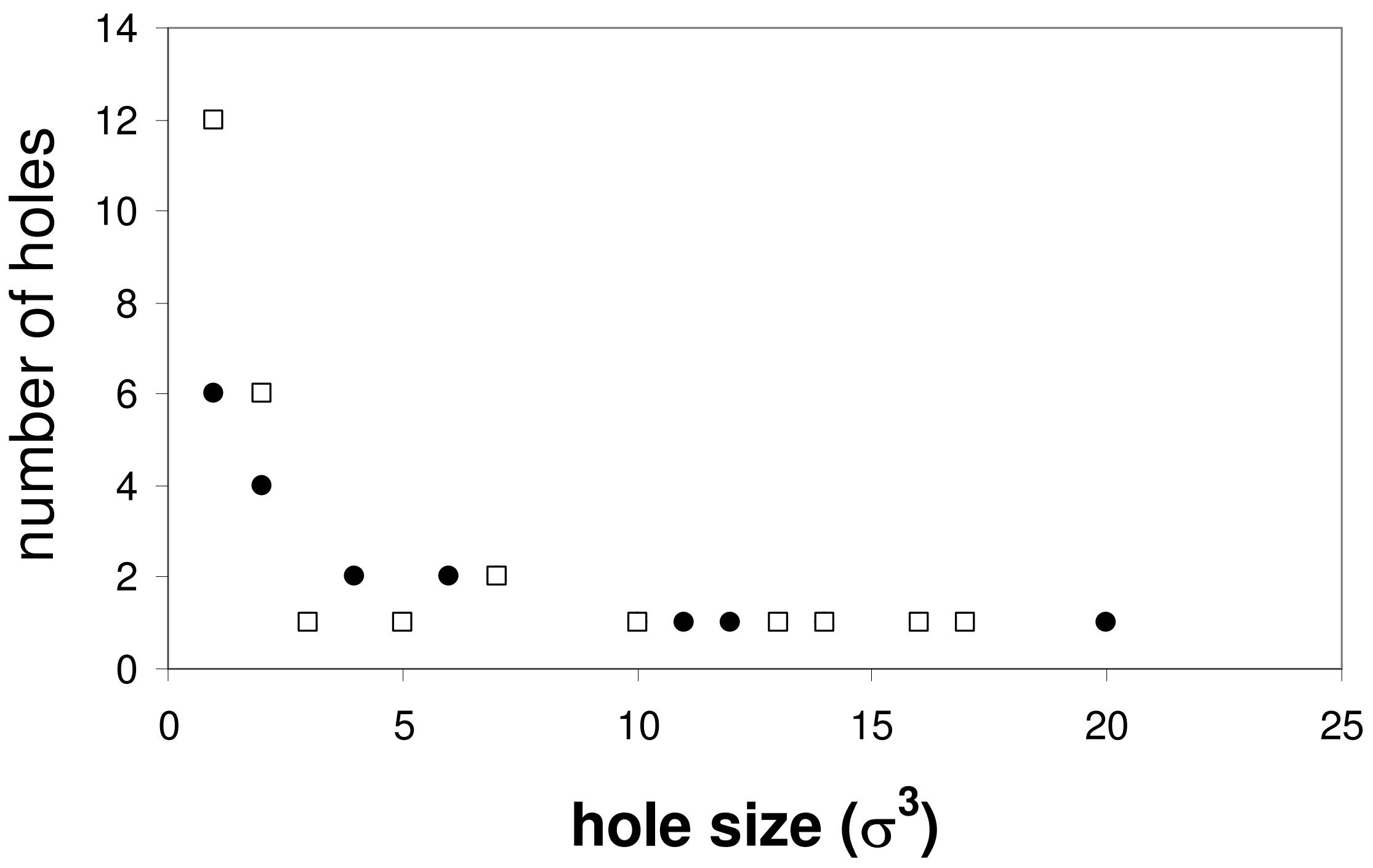

http://mc.manuscriptcentral.com/tandf/jenmol 


\section{Page 27 of 35}

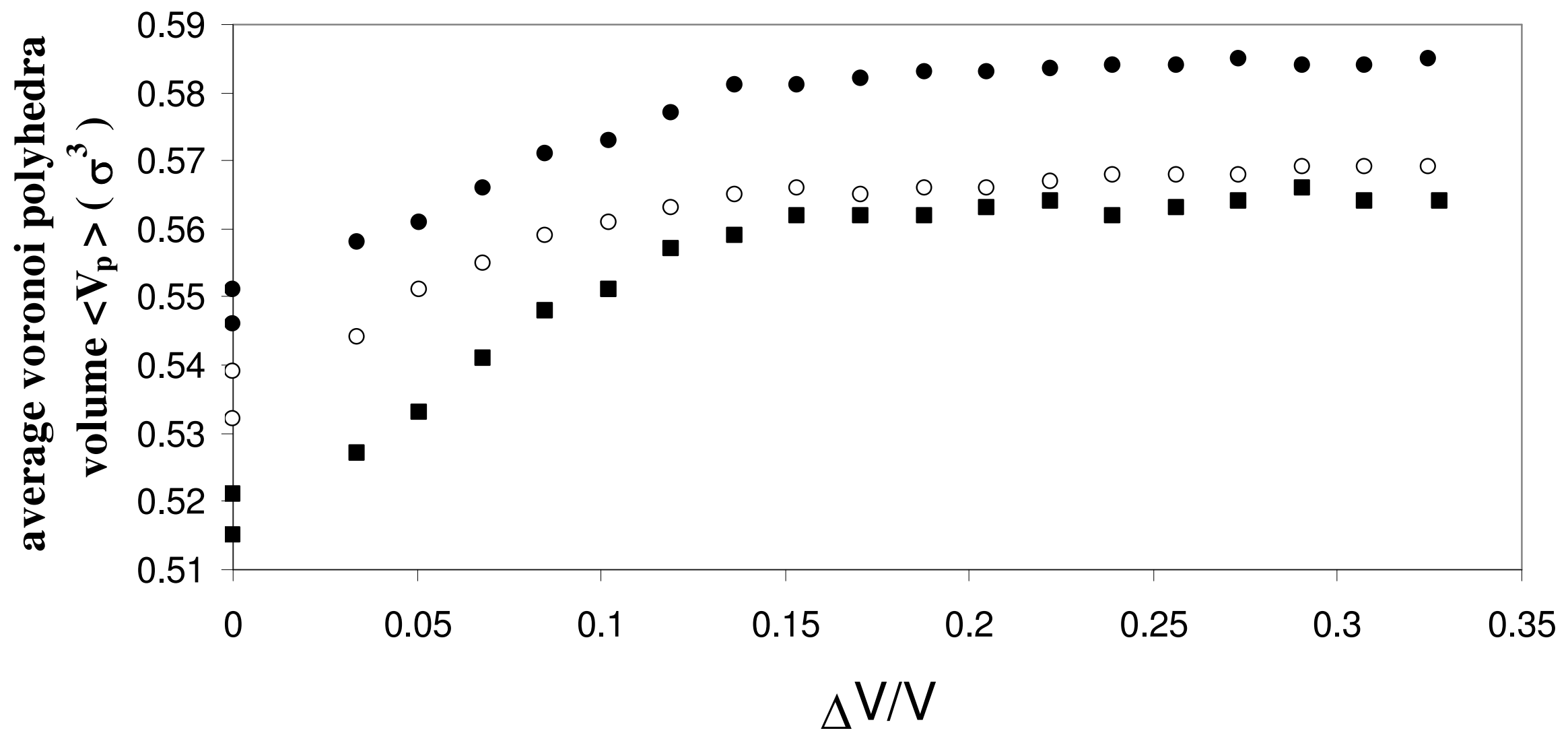

http://mc.manuscriptcentral.com/tandf/jenmol 


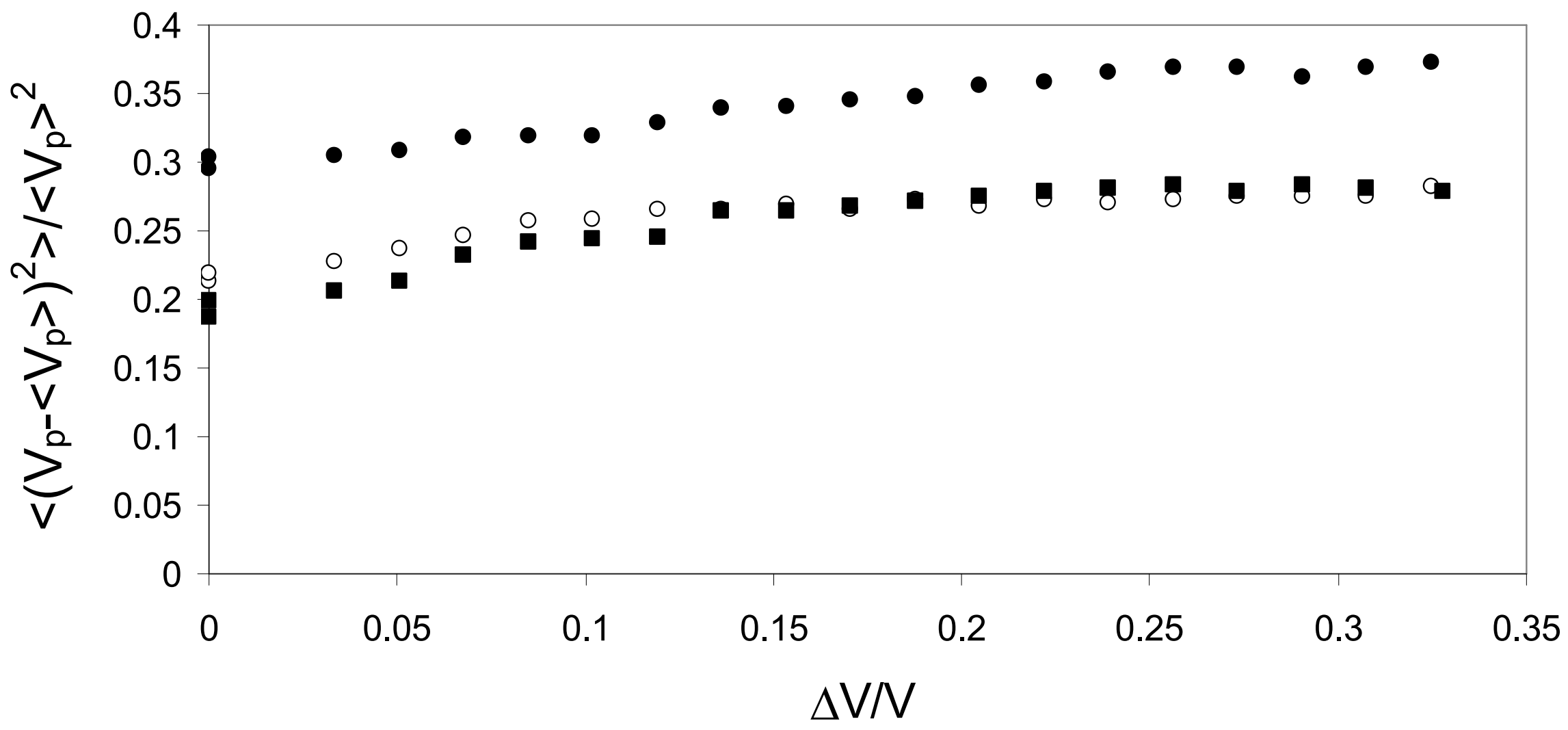




\section{Page 29 of 35}

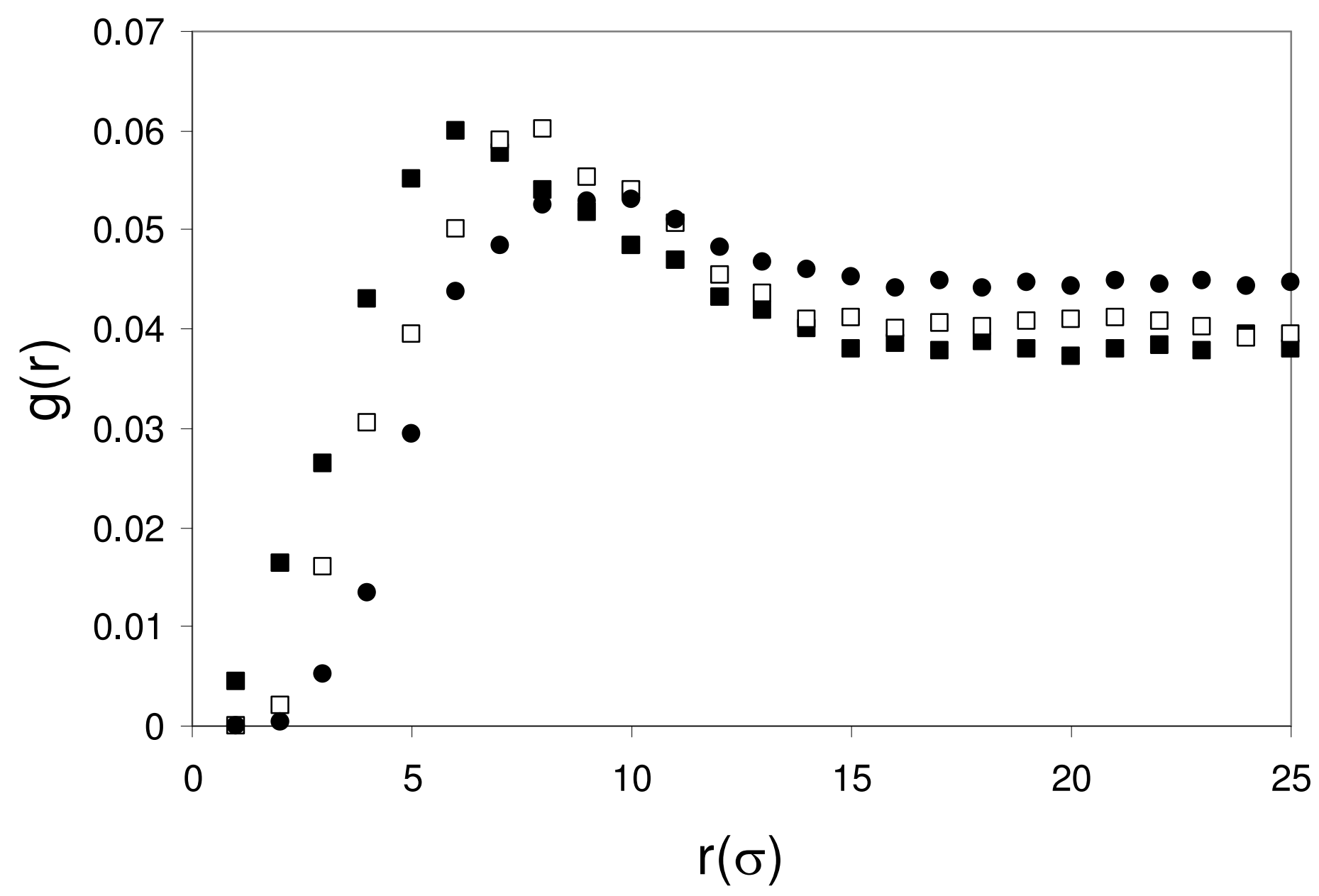

http://mc.manuscriptcentral.com/tandf/jenmol 


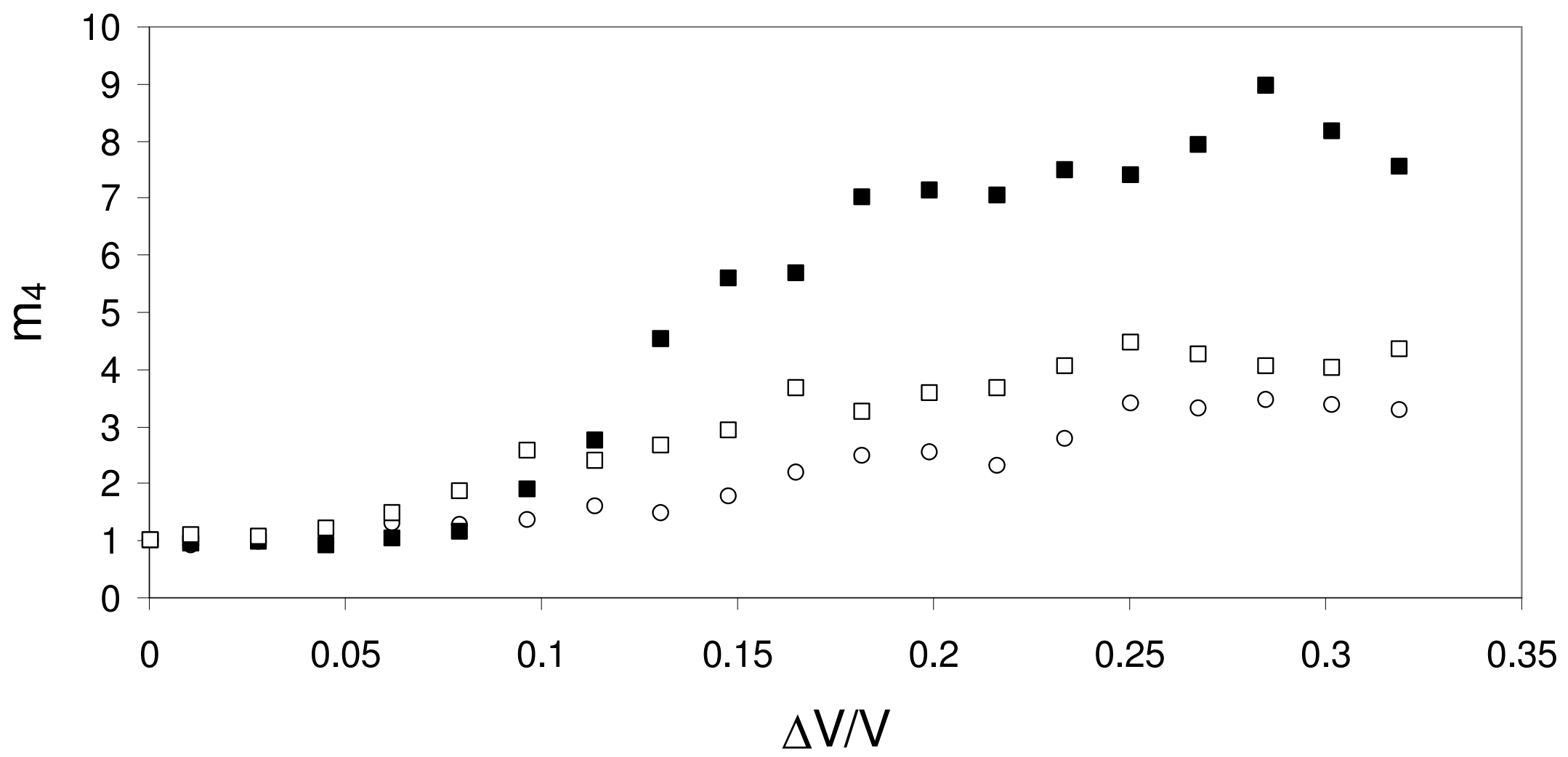

http://mc.manuscriptcentral.com/tandf/jenmol 


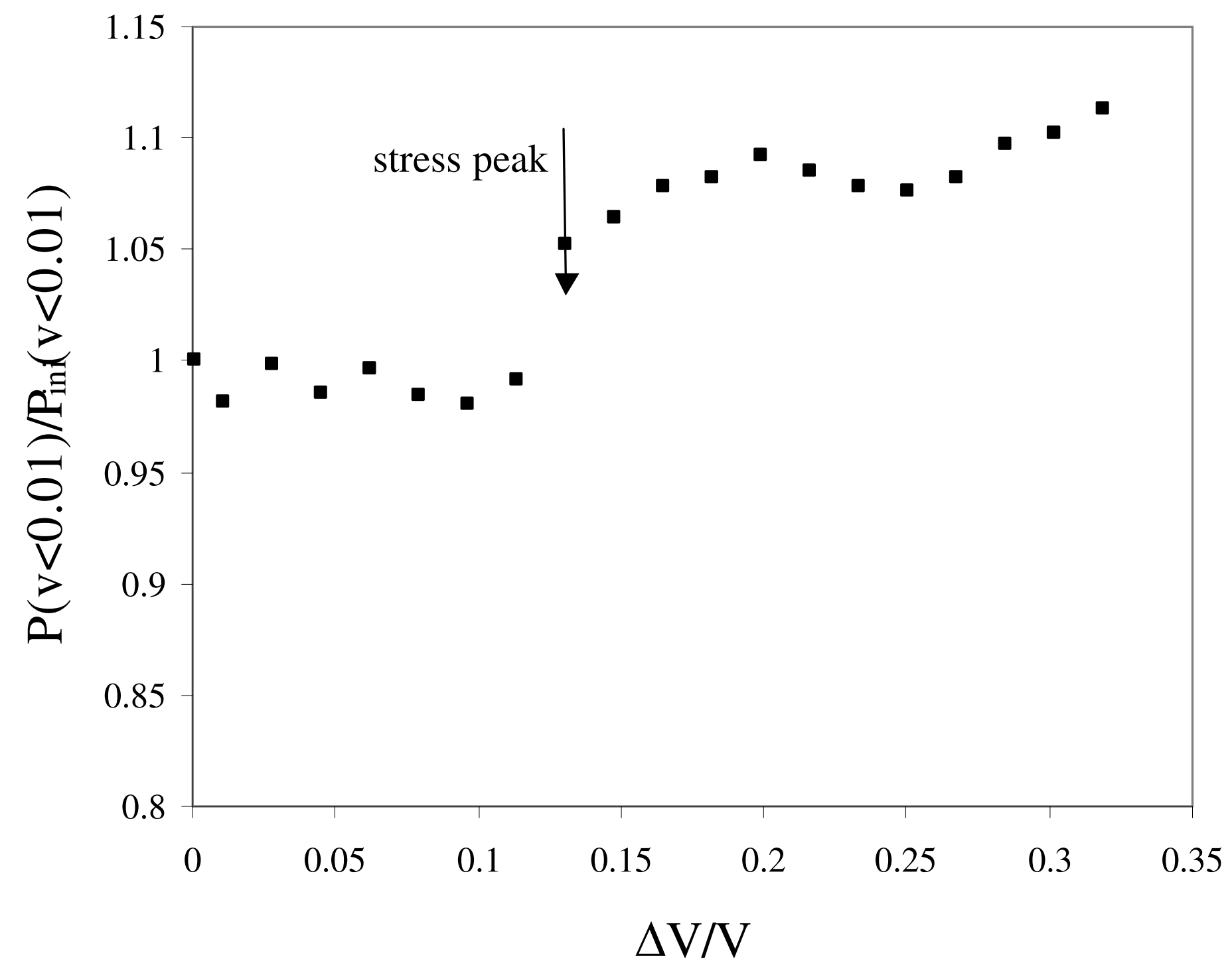




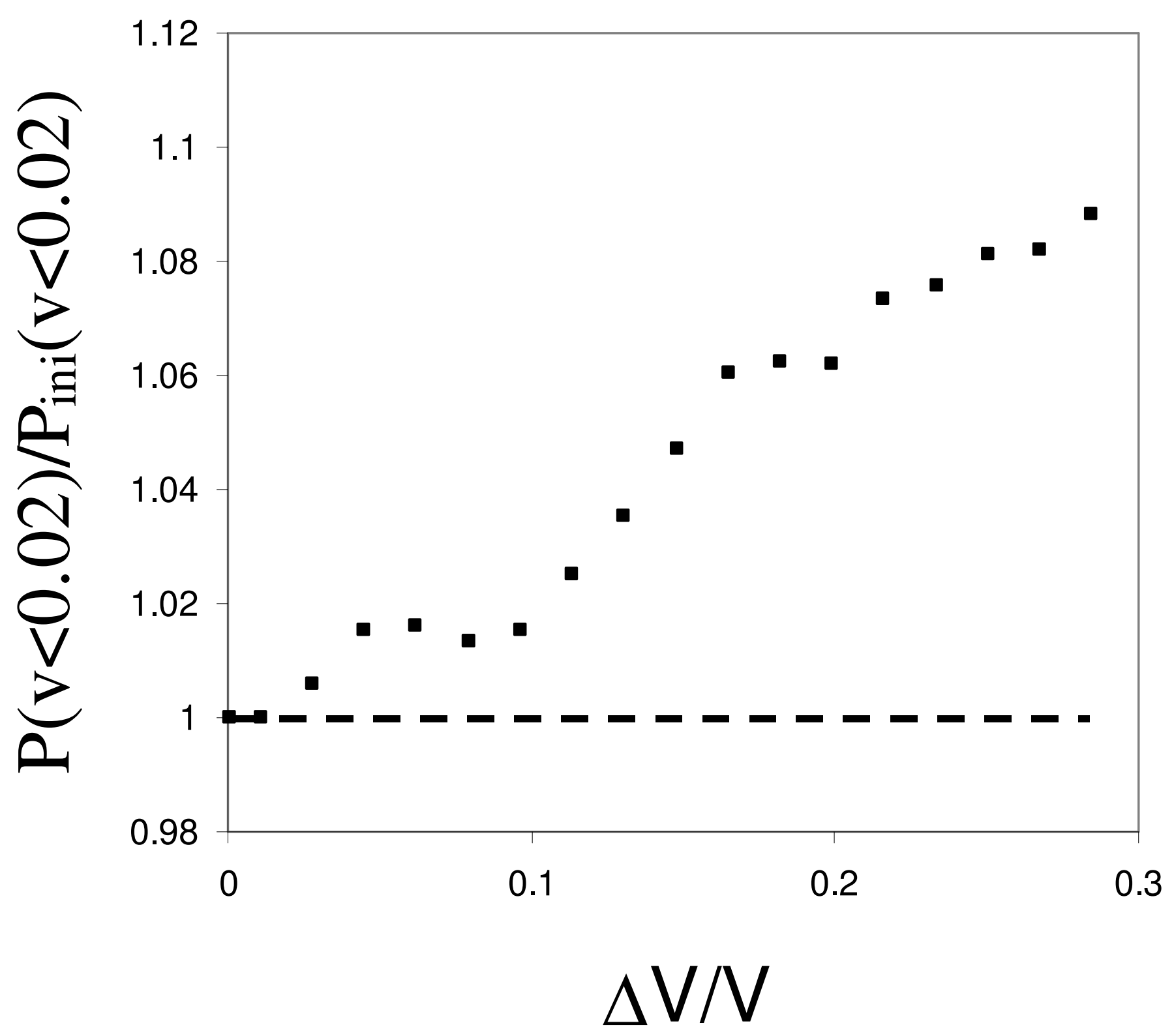

http://mc.manuscriptcentral.com/tandf/jenmol 


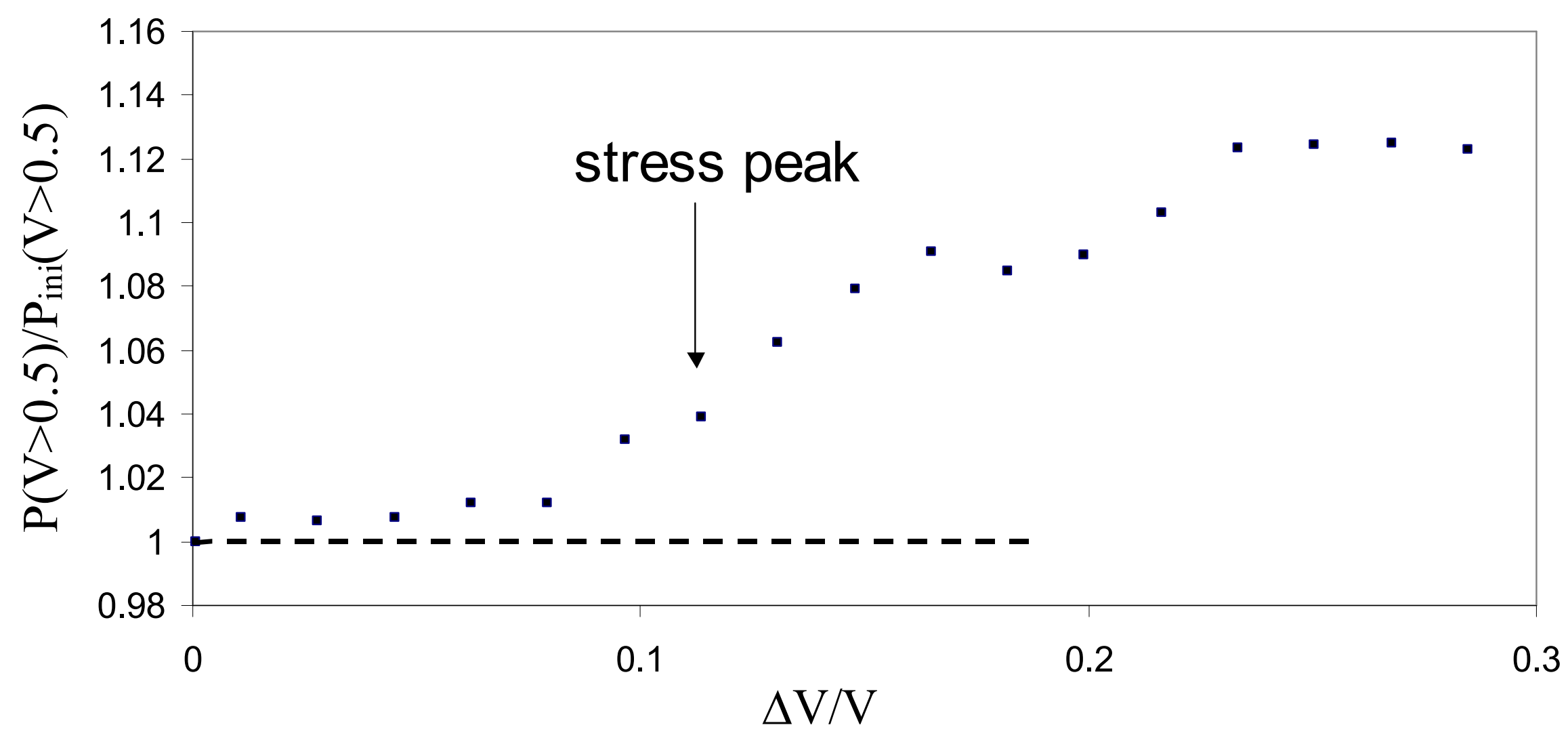




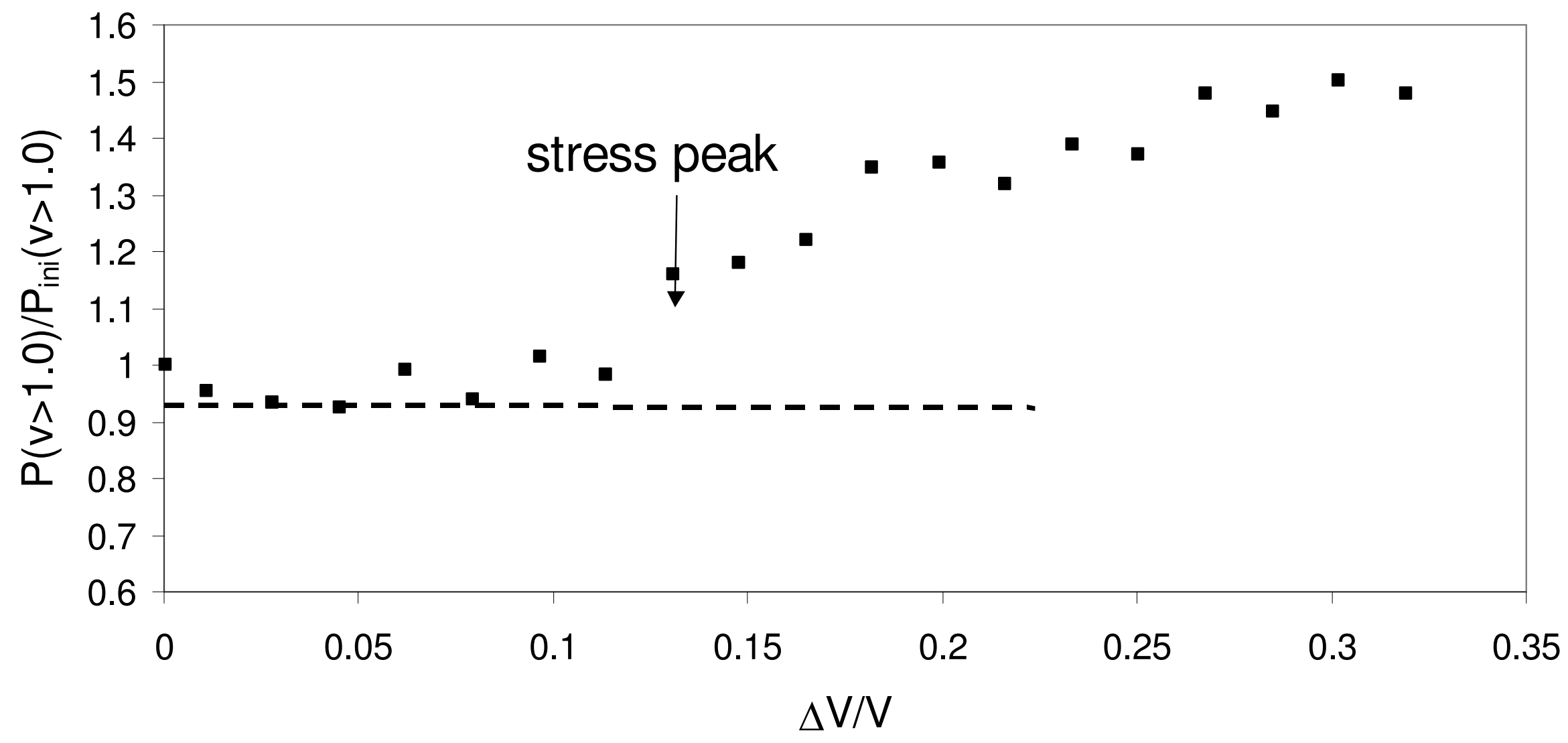




\section{Page 35 of 35}

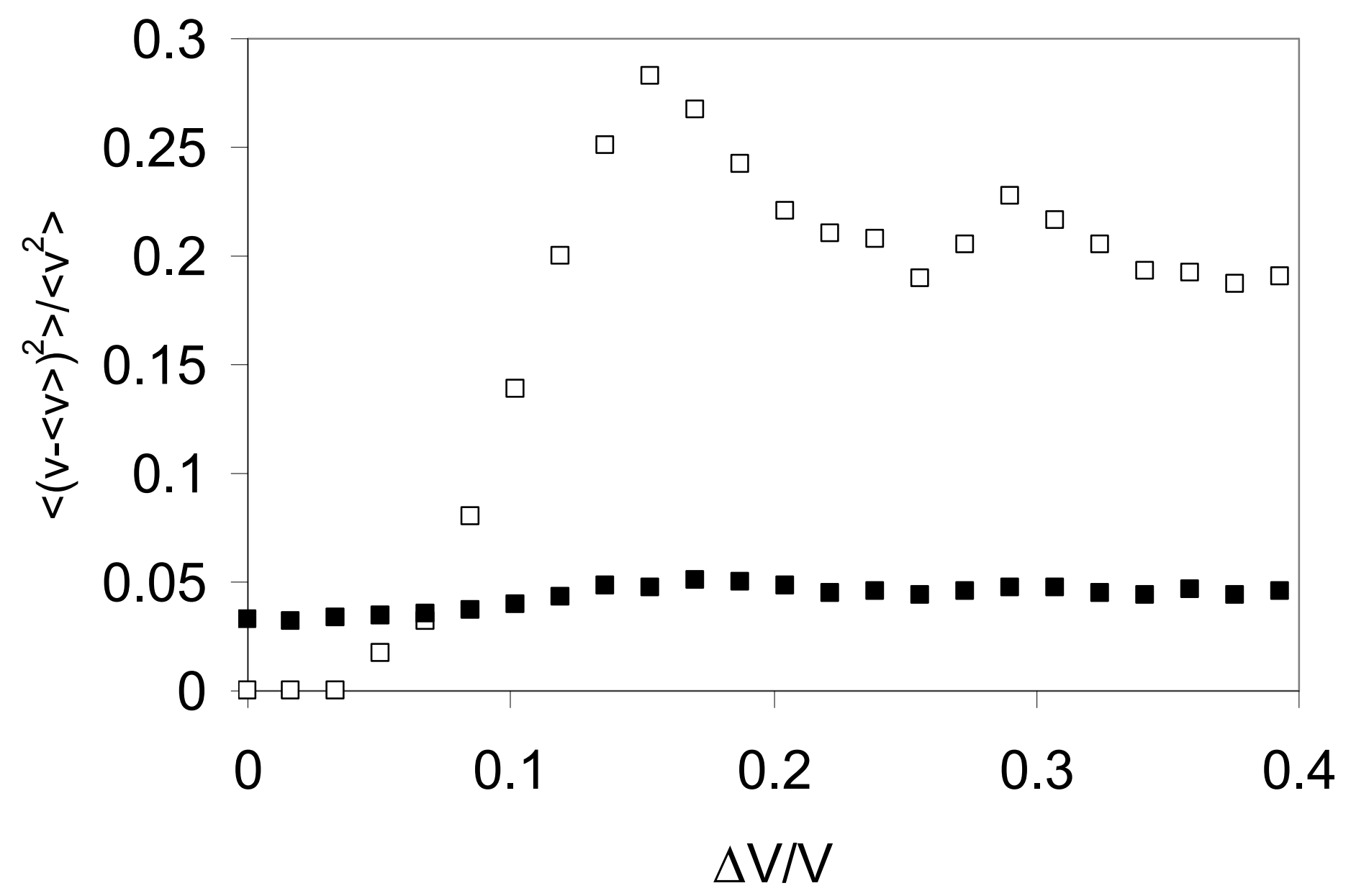

http://mc.manuscriptcentral.com/tandf/jenmol 